\title{
Per riaprire la bottega dei sogni
}

Non passa giorno senza che giunga l'eco di esortazioni volte ad "accendere i sogni", "mettere le scarpe ai sogni","risvegliare i sogni".

Esortazioni dirette per lo più ai giovani che sembrano non disporre più della forza di sognare e alle imprese che senza sogni non possono disporre dell'innovazione necessaria per affrontare la crisi che attanaglia le economie di antica industrializzazione ormai dal 2008.

D'altra parte, come potrebbe nascere e svilupparsi un'impresa in assenza di sogni che sappiano tracciare i contorni della sua ragion d'essere e del ruolo desiderato all'interno della comunità nella quale si inserisce? E chi più dei giovani dovrebbe disporre della capacità di pensare "out of the box" alla ricerca del nuovo?

Così, nel bel mezzo di una crisi che ci accompagna da anni, la parola sogno è tra quelle più citate, quasi possa racchiudere in sé la formula magica per il superamento delle difficoltà attuali.

E non si è lontani dal reale quando si pensa questo. Cosa sta infatti alla base di ogni progresso umano se non un sogno come quello di Cristoforo Colombo, quello di Martin Luther King, di Nelson Mandela, di Adriano Olivetti o di Steve Jobs?

Ecco allora che l'atteggiamento mentale di fronte ai significati di questo sostantivo muta radicalmente anche nel mondo dell'impresa.

Solo pochi anni fa parlare di sogno all'interno delle discipline manageriali sollevava ilarità e silenzi canzonatori. Atteggiamenti che lasciavano spazio ad un finale consolatorio, nel quale si lodavano le belle suggestioni che chi osava parlare di sogni portava sul piatto di una economia che veloce - sempre più in fretta, senza lasciare tempo al pensiero - era lanciata inconsapevolmente verso la sua più grande crisi, "come chi non sa dove va ma ci va di corsa" (Tonino Guerra).

Invero, quando non si ha tempo di pensare a dove si va perché si è troppo impegnati a camminare, $\mathrm{o}$ a correre, è probabile prima o poi inciampare in qualche ostacolo, come ci ricorda Antonin-Dalmace Sertillanges.

Ed eccoci così ad invocare i sogni, ad esortare i giovani a sognare, quasi che il sogno possa nascere da una magica pillola prescritta da un medico specialista di futuro, pillola che ci traghetta in una fantastica epoca dove tutto è possibile e l'innovazione corre libera nelle praterie dell'economia.

Sembra di stare in una favola, ma le favole sono per bambini. Il problema non si pone per i bambini che per parte loro continuano comunque a sognare, il problema si pone per gli adulti che hanno smesso di stupirsi del mondo che hanno intorno e vivono in una paradossale apatia di futuro.

* A Franco Cabras, un caro amico.

sinergie, rivista di studi e ricerche

n. 90, Gennaio-Aprile 2013, pp. VII-XV

ISSN 0393-5108 - DOI 10.7433/s90.2013.01 
E per il mondo degli adulti le favole sono belle e importanti quando si pongono come metafore della vita, non quando diventano una specie di totem, di feticcio, cui affidare il compito di risolvere problemi.

Tutto questo per dire, e ribadire, che i sogni sono una cosa seria e che la morte dei sogni equivale alla morte del progresso, con tutto ciò che la fantascienza potrebbe proiettare nell'ipotesi di una siffatta realtà. Sì, la fantascienza, perché per nostra fortuna non siamo ancora nell'epoca della morte del progresso e solo la fantascienza la potrebbe ora descrivere. Invero, ci sono tutte le condizioni per evitarla se torniamo ad occuparci seriamente di sogni, il che significa ridare spazio ad una fantasia e ad una immaginazione capaci di rigenerare una innovazione diretta a ri-costruire il futuro.

Proviamo a percorrere questa strada dal nostro punto di vista di ricercatori e docenti di management, osservatorio, il nostro, di grande rilevanza perché intercetta il mondo dei giovani e il loro porsi di fronte al mondo che verrà.

Bene, cominciamo con il riflettere sulle facce attonite, ammutolite, spaesate, annoiate, insofferenti, perplesse cui ci si trova spesso di fronte se si chiede a studenti universitari quale sia il loro progetto di vita. È ovvio. Si tratta di un passaggio molto impegnativo. Forse l'epoca della riflessione su un progetto di vita è successiva alla loro età. Chissà? Meglio però lasciare questa questione temporale con il punto interrogativo.

Lasciamo il progetto di vita e parliamo semplicemente di sogni per il loro futuro. Come muta l'atteggiamento dell'aula a questo punto? Incredulità, stupore, si parla di questo in una facoltà di economia? Avversione, perché stiamo perdendo tempo su questioni filosofiche? Vuoto, smarrimento. Come posso sognare se la crisi mi cancella il futuro?

Insomma, per tante ragioni a sfondo prevalentemente sociologico, quindi fuori dalla nostra capacità di approfondimento, abbiamo disabituato i giovani a sognare, forse abbiamo fatto credere loro che tutto quello che si poteva sognare l'avevamo sognato e realizzato noi. E se così fosse non solo avremmo rubato loro i sogni, avremmo rubato loro anche il futuro, in quanto il futuro vive di sogni perché sono questi che alimentano il desiderio di progresso.

Ma di che sogni stiamo parlando? Ovviamente dei sogni ad occhi aperti, quelli che conducono le persone a desiderare, intravedere e progettare un loro ruolo nella società al di là di quello che la realtà e la quotidianità suggeriscono (Francesco Alberoni).

Quei sogni che un tempo si raccoglievano in diari e quaderni gelosamente custoditi in preziosi e personali cassetti, o semplicemente nei pensieri, e che stavano a fondamento delle azioni di ognuno nel desiderio di poter contribuire anche se in parte infinitesimale alla costruzione del futuro di tutti, o se non altro, almeno del proprio.

Oggi quei cassetti sempre più di rado racchiudono sogni. I sogni impauriti da un ipotetico buio avvenire se ne stanno altrove, invisibili però agli sguardi tristi, malinconici, rassegnati, a volte impauriti, ma sempre vuoti di entusiasmo che muovono intorno a loro senza vederli. 
Ma i sogni sono lì, in attesa di qualcuno che dia loro la forza di riprendere forma per camminare e distendere le ali al vento per lanciarsi in volo. I sogni non possono essere soffocati, possono solamente essere tacitati da effimeri atteggiamenti di chi "dai sogni si sente disturbato" (Stanislaw Jerzy Lec).

Cosa si potrebbe proporre per ridare loro forza? Proviamo a rispondere a questa domanda con alcune sintetiche opzioni che poi proveremo a dettagliare nei passaggi successivi. Così potremmo: cercare di capire perché oggi le persone, e i giovani in particolare, sognano poco o non sognano affatto; provare a nobilitare il sogno; analizzare ciò che sta alla base della costruzione del sogno; individuare i tratti del sognatore; valutare le azioni che possono rigenerare la forza di sognare.

Possiamo raccogliere tutto questo in una serie di ulteriori domande che dovrebbero semplificare le riflessioni da sviluppare in merito. Dunque, per ridare forza ai sogni potremmo provare a rispondere a queste domande:

1) Perché le persone sognano poco o non sognano affatto?

2) Cosa significa nobilitare il sogno?

3) Qual è la materia prima dei sogni?

4) È possibile disegnare il profilo del sognatore?

5) Cos'è che impaurisce i sogni impedendo loro di alzarsi in volo?

\section{1) Perché le persone sognano poco o non sognano affatto?}

La domanda non si propone per chi per mestiere sogna come, ad esempio, l'artista o il creativo. La domanda è diretta a chi nel sogno potrebbe trovare un senso per il proprio ruolo nella società indipendentemente da quanto grande esso sia. Per intenderci, un giovane che sta entrando nel mercato del lavoro, un operaio o un impiegato che si trova già nel mondo del lavoro, una persona che si trova nell'età del tempo libero, ma è ancora in forze grazie all'allungamento della durata media di vita. Insomma, il quesito è diretto a capire perché chi vive nella quotidiana normalità sia portato poco a sognare.

È importante sottolineare questo aspetto perché la forza del sogno non dipende solo dalla presenza di pochi grandi sogni, ma dalla diffusione di tanti piccoli sogni che possono costellare il cambiamento quotidiano. In realtà, i sogni dei bambini nascono grandi, i sogni degli adulti non sempre seguono questa via e spesso diventano grandi dopo essere stati piccoli e coltivati con tenacia e caparbietà.

Detto questo, proviamo a individuare almeno alcune delle ragioni che portano una moltitudine di persone a rinunciare al sogno.

Si rinuncia a sognare perché:

- il sogno è un lusso che non ci si può permettere a fronte di una situazione economica che porta a concentrare tutte le energie sul come governare il ménage quotidiano;

- un tempo si aveva la consapevolezza che ci fosse il futuro quando oggi si è portati a pensare che non ci sia più per la tendenza alla visione breve, al tutto e subito e alla dilatazione del presente (Marc Augé); 
- nella società ciò che viene riconosciuta e premiata è l'azione a scapito del pensiero, l'efficienza a scapito dell'efficacia e dell'innovazione, la velocità a scapito dell'osservazione e della riflessione;

- essere sognatori è visto come un difetto, viene visto come una fuga dalla realtà e non si ha il coraggio di sopportare questa, più o meno, velata critica;

- ci si rifugia nei mondi paralleli creati dalle moderne tecnologie della comunicazione abdicando alla costruzione del pensiero.

Ovviamente, altri motivi ci saranno, ma questi sono quelli incontrati più diffusamente nel nostro cammino di studiosi e docenti di management.

Va da sé che non tutte le ragioni citate si sommano nella stessa persona. Anche se in taluni casi questo può accadere, in altri può ricorrere anche uno solo dei motivi citati. Basta la presenza di uno solo di questo, infatti, per portare la persona a disperdere la capacità di sognare.

\section{2) Cosa significa nobilitare il sogno?}

La risposta a questa domanda chiama direttamente in causa una delle ragioni elencate nella lista dei perché le persone sognano poco o non sognano per nulla: il fatto che nella società l'essere sognatori sia visto come un difetto.

Quante volte ci siamo sentiti dire, o abbiamo sentito dire a qualcun altro, "non stare lì a sognare, torna con i piedi a terra"?

Nascosta dietro questa esortazione sta il convincimento che il sognatore, il visionario, sia una persona che perde tempo, tempo che non può essere buttato in questo modo perché andrebbe a scapito dell'efficienza.

Curioso è il fatto che negli studi di strategia d'impresa ben si precisa sempre per dirla all'inglese -, che "the vision and mission of a company should be a driving force, setting the strategic direction of the business".

E allora? Perché ai sognatori, ai visionari, a chi vede ciò che ancora è nascosto, a chi va al di là dei vincoli del reale della propria quotidianità per elevarla con uno sguardo al futuro, a chi pensa fuori dagli schemi non deve essere dato credito?

Perché "chi sgrana gli occhi nello sforzo di sognare, chi cerca di guardare oltre al contingente è presto giudicato: è appunto un visionario. La sua è una visione distorta della realtà, le sue idee sono prive di riscontro, i suoi progetti sono campati in aria"? (Francesco Varanini).

Perché "i sogni di qualcuno non faranno dormire gli altri”, ci risponderebbe il già richiamato Stanislaw Jerzy Lec.

D'altra parte cosa sta a fondamento dell'essere imprenditore se non il sogno? La parola imprenditore sappiamo deriva dal verbo imprendere che a sua volta significa fare cose nuove, ma come si possono fare cose nuove se non si forza la realtà per vedere oltre a ciò che è noto e ormai acquisito?

Nel management di questo si parla poco, lo si accenna a mala pena, perché il management deve muovere per oggettività, per misurazione, per razionalità. L'immaginazione, che, come ricorda Albert Einstein, è più importante della conoscenza perché abbraccia il mondo, spesso non viene nemmeno menzionata 
anche se la non razionalità di simoniana memoria ben apriva la strada in questa direzione.

Forse lasciare negli studi di management uno spazio maggiore per la storia dell'imprenditorialità portando la copiosa documentazione che esiste sui sogni di imprenditori quali Adriano Olivetti, Henry Steinway ed Henry Heinz potrebbe contribuire a nobilitare questa parola e rendere evidente che ogni progresso e la costruzione del futuro passano proprio di lì. Come pure, in generale, recuperare il valore di una parte della letteratura spesso considerata di serie b, come si usa dire, quale la letteratura del fantastico che ha saputo anticipare numerose delle acquisizioni di oggi. O ancora osservare il frutto dell'arte che sempre sa cogliere in anticipo ciò che nella società si sta formando o che esplicita mirabilmente le connessioni tra sogno e realtà, come avviene con la mirabile Promenade di Marc Chagall, dove il pittore con i piedi ben saldi a terra è proiettato verso l'alto dalla moglie che si libra in volo in una strabiliante metafora dell'equilibrio razionalitàsogno.

3) Qual è la materia prima dei sogni?

I sogni rivelano tutta la loro importanza se si considera che li si può vedere come un territorio senza confini nel quale germogliano le idee, siano esse grandi o piccole, rivoluzionarie o semplicemente volte al miglioramento di ciò che già si fa e si conosce.

Se le idee stanno al sogno, il sogno a cosa sta? In altre parole, se la materia prima delle idee è rappresentata dal sogno, quale sarà la materia prima del sogno?

In proposito si può dire che il sogno sia la risultante di una complessa alchimia nella quale entrano diverse componenti.

Prima fra tutte la fantasia, ossia la capacità di vedere ciò che è invisibile alla realtà, la capacità di intravvedere ed esplorare l'impossibile sino ad immaginarlo per poi trasformarlo in un'idea da valutare nella sua fattibilità concreta.

$\mathrm{Ne}$ fa certo parte il coraggio che consente di uscire dagli schemi conosciuti anche in opposizione al senso comune.

Anche l'umiltà è parte di questa alchimia perché il nuovo può forgiarsi solo a partire dalla consapevolezza dei limiti dai quali si muove e della possibilità di miglioramento continuo nella proiezione verso i traguardi desiderati.

La curiosità non può mancare perché è ad essa che si deve la ricerca delle forme per procedere sempre oltre ciò che già è acquisito.

Il sogno non può, infine, nascere in assenza di entusiasmo, ossia di quello slancio e di quella passione che forniscono l'energia capace di credere in un progetto futuro al di là delle circostanze avverse e degli ostacoli che apparentemente potrebbero soffocarlo sul nascere.

Considerando che i sogni portano all'idea e tramite essa all'innovazione, la quale a sua volta è connessa al cambiamento, condizione questa che incute timore in chi desidera mantenere lo status quo, il percorso dei sogni non può che essere costellato di ostacoli per superare i quali è necessario disporre della necessaria tenacia. 
Se gli ingredienti indicati sino a questo punto attengono alla sfera personale, cioè ai tratti dell'individuo, vi sono almeno due condizioni di contesto la cui presenza crea la possibilità perché l'alchimia del sogno possa avviarsi.

Si tratta, da una parte della speranza, dall'altra della fiducia.

La speranza come contesto si rileva quando l'ambiente nel quale ci si muove consente il formarsi della forza di guardare avanti anche quando sembra non ci sia nulla più da vedere. Il che significa disporre di una speranza attiva nell'individuo che non vive una passiva attesa di quello che si spera avvenga, ma muove in forme intraprendenti nella direzione della costruzione del mondo desiderato.

D'altra parte, la percezione delle dotazioni di fiducia disponibili nel contesto in cui si agisce promuove quel senso di affidamento che accresce la forza di pensare a lungo termine, con lo sguardo ad orizzonti lontani, nella consapevolezza di poter contare su altre energie diffuse nel territorio o nella rete di relazioni cui si partecipa.

4) ¿̀ possibile disegnare il profilo del sognatore?

Nonostante - come rilevato a proposito della necessità di nobilitare il visionario ed il sognatore - sia diffusa l'opinione che il sognatore "sia quella persona che sta sempre con la testa per aria, quello che perde tempo", la risposta a questa domanda non può che essere un sonoro sì, è possibile, scoprendo anche che il visionario è tutt'altro che una persona con la "testa sempre per aria".

$\mathrm{Al}$ riguardo è forse opportuno sottolineare che il sogno di cui stiamo parlando è in sé molto oggettivo. È quello che introduce all'idea e per questa via alla creatività, il cui percorso non si ferma alla formulazione dell'idea, ma arriva alla sua concretizzazione di qualunque tipo di idea si tratti. Concretizzazione che spesso passa attraverso reiterati tentativi andati a vuoto, mai però capaci di indurre l'abbandono del percorso intrapreso. E la storia di Edison e dei suoi innumerevoli tentativi per arrivare alla lampadina sta lì a sottolinearlo.

Dunque, "sognare non è fuggire dalla realtà. È sganciarsi dalle evidenze, lasciare deliberatamente i sentieri dell'obbedienza, proiettarsi in una realtà che si osa pensare differente (Riccardo Petrella).

Il sognatore, invero, non può prescindere da una sua razionale necessità di agire, il sognatore si sente parte del mondo, non desidera affatto porsi al di fuori di esso. Il sognatore non è un asceta.

Dunque, è possibile disegnare un profilo del sognatore, cioè di colui che desidera forzare il reale per pervenire a qualcosa di nuovo.

Posto che gli aspetti richiamati a proposito della materia prima dei sogni fanno parte a tutti gli effetti del profilo del sognatore che quindi deve essere umile, curioso, coraggioso, tenace e dotato di fantasia, oltre che possedere la forza di credere nel futuro (speranza) e nelle relazioni tra individui e organizzazioni (fiducia), esistono altri caratteri che rivelano i tratti del sognatore.

Eccoci qui allora a proporre una lista costruita sulla base dell'osservazione di alcune esperienze aziendali quali Bonotto, impresa tessile del vicentino, Pedrollo, impresa meccanica del veronese, Davines, impresa parmense del settore della cosmesi, Cucinelli impresa umbra operante nel settore dell'abbigliamento, ma anche 
dell'esperienza raccolta in anni di letture e visioni di film vicini o anche apparentemente distanti dall'impresa, oltre che nelle relazioni con imprenditori, manager e studiosi di azienda.

Così riteniamo di non essere molto lontani dal reale nel sottolineare che il sognatore si muova intorno a questi caratteri:

- desidera ricercare un proprio posto nella società;

- produce continuamente domande per esplorare il campo nel quale si trova ad agire;

- non accetta il vincolo dell'impossibile e sa convivere con il rischio;

- sa che il presente è un ponte che unisce il passato al futuro desiderato;

- raccoglie nella sua giornata un tempo per pensare associato a quello per agire;

- prova diletto nel lavoro che fa e sa "attaccare" il proprio sogno ad altri;

- apprezza il silenzio;

- sa quando è il momento di allontanarsi dal computer e dalla rete;

- ama l'arte per la sua capacità di ispirare e di disarticolare il pensiero;

- è aperto alla diversità;

- preferisce chiedersi "perché no?" piuttosto che "perché?";

- vive il cambiamento con il fervore della ricerca del nuovo;

- crede nella fantasia e la sa tradurre in immaginazione e idee;

- il denaro non è un suo obiettivo.

5) Cos'è che impaurisce i sogni impedendo loro di alzarsi in volo?

Ciò che impaurisce i sogni è il senso che viene associato ad una delle dimensioni del tempo: il presente. Cerchiamo di capire perché.

Ben sappiamo che il tempo è nella nostra mente distinto nelle sue tre componenti di passato, presente e futuro. Ben sappiamo anche che solo il presente è in sé reale, perché sia il passato che il futuro sono costruzioni mentali nate sull'asse immaginario di un tempo senza interruzioni. Il passato, infatti, è memoria, storia, il futuro è ipotesi, possibilità. Nulla è dato compiere sul passato, se non nei termini di una sua ricostruzione interpretativa e della consapevolezza acquisita sulla base dell'esperienza. Tutto è possibile fare per il futuro, perché il futuro è ancora plasmabile attraverso ciò che si può produrre nel presente.

Ecco, i sogni impauriti stanno nascosti perché sono perseguitati da un presente dilatato che non pensa al futuro, un presente ripiegato su se stesso incapace di alzare lo sguardo sul tempo che verrà, perché lanciato in una forsennata corsa del tutto $\mathrm{e}$ subito in omaggio ad una cultura della sicurezza che disperde il senso del rischio al quale sono tanto affezionati i sogni.

Viviamo, infatti, in una società forgiata dal mercato, nella quale gli individui frugano forsennatamente nel presente alla ricerca di una felicità, fondata su un'ingombrante presenza di consumi indotti dal passaggio da un'economia del bisogno ad un'economia del desiderio, ormai approdata ad una vera e propria economia del capriccio. Capriccio sapientemente sostenuto da uno spietato apparato comunicativo che fa assumere alle proposte di mercato i tratti dell'essenzialità agli occhi del consumatore, attraverso una pubblicità che sa abilmente unire "l'inutile al 
dilettevole" (Ennio Flaiano). Condizione questa che ammalia l'individuo portandolo ad inseguire il nuovo capriccio quasi indipendentemente dalla propria volontà, in una sorta di ipnosi da consumo nella quale le persone appiattiscono il proprio pensiero in un "eterno e allucinato presente", perpetrando un vero e proprio "furto di futuro" (Marc Augé), in una dilagante logica di "pensiero breve".

Comportamento che ha avuto un ruolo non secondario nella generazione della crisi con la quale stiamo convivendo ora, nel 2013, da almeno 5 anni. Crisi che, per parte sua, ha allontanato ulteriormente le persone dal futuro diffondendo in molti un'ansia per il tempo che verrà, al punto che nitida e palpabile si intravvede serpeggiare nella comunità la paura di ciò che il domani potrà portare. Quasi che il domani non dipendesse da quello che ognuno fa per la parte che gli compete, o gli competerebbe.

Ecco quindi cosa occorrerebbe fare per ridare slancio ai sogni: occorrerebbe ridare un senso al presente popolandolo di speranza e di fiducia, per ridare forza al desiderio di costruire e non solo consumare e distruggere.

Forse a questo punto abbiamo fatto un passo avanti, o forse no. Ammettendo almeno per un momento di averlo fatto, occorre capire come poter agire per realizzare questo cambiamento di senso.

Quattro potrebbero essere le direttrici da tener presente:

- $\quad$ non disperdere la speranza: la speranza non può essere soffocata da una crisi per quanto ampia questa sia, la speranza non è stata soffocata dal totalitarismo della prima metà del Novecento e dagli anni di piombo della seconda metà del Novecento (Federico Rampini); il futuro non è stato cancellato e non sarà mai cancellato, la crisi può essere un potente motore di cambiamento nella direzione della consapevolezza individuale che il mercato, nelle sue forme attuali, ha rivelato il suo fallimento trasformandosi in una sorta di totalitarismo economicofinanziario;

- rafforzare la fiducia in sé nella consapevolezza che ci si può reinventare se si inizia a guardare intorno con curiosità e attenzione per cogliere anche le piccole cose che possono consentire di valorizzare la conoscenza e l'esperienza dei singoli;

- agire individualmente nella direzione del rispetto e del dono frugando negli innumerevoli problemi che passano accanto per trovare in essi le opportunità che gelosamente sempre racchiudono al loro interno in una sfida continua nella direzione del progresso;

- essere consapevoli che gli ostacoli che si incontrano nel cammino sono il risultato del modo con cui si legge la realtà e che quando i problemi appaiono insormontabili il guardarli da una prospettiva diversa consente di trovare i percorsi che conducono alla loro soluzione.

Tutto questo, consci del fatto che "un sogno sembra solo un sogno fino a quando non si comincia a lavorarci. E allora può diventare qualcosa di infinitamente grande" (Adriano Olivetti).

Forse a questo punto saremo in grado di riaprire "la bottega dei sogni". 
"When people believe in boundaries they become part of them", Don Cherry, Jazz Musician, ripreso da Change This, Josh Linkner, "Disciplined Dreaming", february, 2011.

\section{Postilla}

Sappiamo bene che, al di là di tutto quello che possiamo scrivere o pensare, la realtà propone momenti nei quali ci si chiede perché continuare, o perché provare, a sognare. Momenti nei quali il mondo sottosopra sembra chiudere definitivamente allo sguardo entusiastico diretto a progetti del futuro. Momenti nei quali il mondo spinge l'individuo nella direzione della chiusura in sé e in un rassicurante presente, tale solo perché è vicino. Ecco, per questi momenti non ci sono riflessioni risolutive generalizzabili, ci può però essere l'invito a non dimenticare mai che dietro le nuvole c'è sempre il sole e che "il sole è nuovo ogni giorno" (Eraclito).

Claudio Baccarani

Gaetano M. Golinelli 
